\title{
Evaluando el impacto de la implantación de la calidad en la Administraciones Públicas. Las Cartas de Servicios en la Comunidad de Madrid
}

\author{
Jorge Crespo González \\ Departamento de Ciencia Política y de la Administración II \\ Universidad Complutense de Madrid \\ J. Ignacio Criado \\ Departamento de Ciencia Política y de la Administración II \\ Universidad Complutense de Madrid
}

\begin{abstract}
Sumario: 1. INTRODUCCIÓN. 2. LAS CARTAS DE SERVICIOS COMO HERRAMIENTAS DE TRANSFORMACIÓN EN LA GESTIÓN PÚBLICA. 3. RESULTADOS SOBRE EL CAPITAL HUMANO. 3.1. La participación de los colectivos de personal en la formulación, implantación y evaluación de las Cartas de Servicios. 3.2. La influencia de la introducción de las Cartas de Servicios sobre las actividades de gestión de personal. 4. RESULTADOS SOBRE LOS RECURSOS MATERIALES Y TECNOLÓGICOS. 4.1. Futuras dotaciones presupuestarias en torno a las Cartas de Servicios. 4.2. La mejora de los recursos humanos, materiales y tecnológicos. 4.3. Las tecnológias en las Cartas de Servicos. 5. REFLEXIONES SOBRE LAS CARTAS DE SERVICIOS Y SUS RESULTADOS. 5.1. Balance específico: las Cartas de Servicios y los recursos disponibles. 5.2. Balance general: el impacto de las Carta de Servicios en la Administración de la Comunidad de Madrid: entre la continuidad y el cambio. 6. CONCLUSIONES.
\end{abstract}

Resumen: Dentro del contexto de transformación y modernización de los aparatos públicos de los distintos países de la OCDE, la aparición de la calidad marcó un hito a principios de los años 1990 en la gestión de las organizaciones públicas, generándose un conjunto de herramientas con el objetivo de poner en el eje central de la acción pública las necesidades de los ciudadanos y su satisfacción. Una de las herramientas más relevantes son las Cartas de Servicios. Las Cartas de Servicios constituyen uno de los instrumentos de mejora de la gestión más utilizadas por las administraciones públicas españolas durante los últimos años, siendo la Comunidad de Madrid uno de los casos pioneros en su adopción e implantación. Este trabajo presenta parte de los resultados obtenidos a través de un proyecto de investigación, financiado por la Comunidad de Madrid, en el que se analizaron las Cartas de Servicios operativas dentro de sus unidades administrativas, así como el impacto en ellas de su implantación, aunque se centra en el ánalisis de dos dimensiones de la gestión pública: el capital humano y los recursos materiales. Con todo ello se extraen determinadas conclusiones generales sobre la aportación de estos instrumentos en el buen gobierno y la gestión pública.

\section{INTRODUCCIÓN}

Las Cartas de Servicios (CdS) constituyen uno de los tipos de herramientas de mejora de la gestión más utilizadas por las administraciones 
públicas españolas durante los últimos años, siendo la Comunidad de Madrid (CAM) uno de los casos pioneros en su adopción e implantación. En las siguientes páginas se presentan parte de los resultados obtenidos a través de un proyecto en marcha sobre la Administración de la CAM, en el que se analizaron todas las CdS operativas dentro de sus unidades administrativas, así como el impacto en ellas de su implantación ${ }^{1}$. En concreto, este artículo se centra en el análisis de dos dimensiones de la gestión pública: el capital humano y los recursos materiales.

El nacimiento de las CdS se inscribe dentro del contexto de transformación y modernización de los aparatos públicos de los distintos países de la OCDE. Muchos de los cambios recientes en las Administraciones públicas se han inspirado en la Nueva Gestión Pública, como tendencia administrativa que se caracteriza por integrar un conjunto de técnicas y herramientas susceptibles de mejorar la eficacia y la eficiencia del sector público en un momento de gran exigencia por parte de los ciudadanos. Dentro de esa dinámica de cambio, la aparición de la calidad marcó un hito a principios de los años 1990 en la gestión de las organizaciones públicas, generándose un conjunto de herramientas con el objetivo de poner en el eje central de la acción pública las necesidades de los ciudadanos y su satisfacción. Una de las herramientas más relevantes son las CdS.

A pesar de la difusión de las $\mathrm{CdS}$ en diferentes contextos político-administrativos y que en la esfera comparativa existe un nutrido elenco de estudios sobre $\mathrm{CdS}^{2}$, en el caso español las aportaciones son mucho más esca$\operatorname{sas}^{3}$. Teniendo en cuenta estas limitaciones sobre el ámbito español, esta investigación se propuso analizar el impacto de la introducción de las $\mathrm{CdS}$ sobre la mejora de los servicios públicos en la Administración de la Comunidad de Madrid utilizando diferentes fuentes de información, particularmente empíricas ${ }^{4}$. El objetivo principal consistió en analizar los

\footnotetext{
${ }^{1}$ Esta investigación fue desarrollada con el apoyo de la Dirección General de Investigación de la Comunidad de Madrid (Proyecto Ref. 06/0164/03).

2 En el ámbito británico destacan los trabajos pioneros de Bynoe (1996), Chandler (1996), Willet (1996), pero los análisis también se han realizado sobre otros contextos como India (Sharm y Agnihotri, 2001), Italia (Schiavo, 2000) o Australia (McGuire, 2001).

${ }^{3}$ Se trata de textos descriptivos, evaluaciones internas o documentos oficiales, mientras las aportaciones académicas o evaluaciones desde el ámbito de la Ciencia de la Administración son mucho más escasas: ver Olías de Lima (2003).

${ }^{4}$ El trabajo de campo consistió en la aplicación de un cuestionario enviado a todas las unidades y servicios de la Administración de la Comunidad de Madrid que disponían de una CdS en el momento de la realización de la investigación, así como la realización de entrevistas en profundidad a una muestra del conjunto de aquéllos. Todo ello, se complementó con el acceso a documentación referida a las CdS en la Comunidad de Madrid, así como una exhaustiva revisión bibliográfica.
} 
resultados de la aplicación de este instrumento desde el punto de vista intraorganitivo utilizando como dimensiones de estudio los diferentes factores organizativos (diseño orgánico, recursos humanos, modos de gestión, recursos materiales y tecnológicos, información y comunicación). Este trabajo ofrece las conclusiones sobre dos de esos factores, los recursos humanos y los recursos materiales y tecnológicos, ya que son un exponente crítico del alcance de los cambios en los servicios a propósito de la introducción de las CdS.

El análisis que se presenta se divide en las siguientes partes. La primera ubica las CdS en tanto herramientas de transformación en la gestión pública. En la segunda y tercera partes se presentan de forma detallada los resultados derivados de la introducción de las CdS sobre el capital humano y los recursos materiales y tecnológicos, diferenciando un conjunto de indicadores sobre cada una de esas dimensiones, lo que constituye el eje central de este trabajo. En la cuarta parte, se realizan unas reflexiones sobre las CdS y sus resultados a la luz de los datos empíricos de la investigación. Finalmente, se cierra este estudio con una conclusión que sintetiza los contenidos principales del trabajo y abre una línea de trabajo para el futuro.

\section{LAS CARTAS DE SERVICIOS COMO HERRAMIENTAS DE TRANSFORMACIÓN EN LA GESTIÓN PÚBLICA}

En nuestro entorno político-administrativo, los Estados están ocupados de forma creciente en la construcción de capacidad en sus propios aparatos públicos para afrontar los retos del presente y los que se avizoran en el futuro. En ello consiste esencialmente los procesos de modernización administrativa, que pueden culminarse con medidas de diversa naturaleza y procedencia, como las que preconiza la Nueva Gestión Pública (NGP).

La NGP, que acusa influencias de diversas escuelas de pensamiento que van del gerencialismo clásico a la economía de las organizaciones, pasando por la Teoría de la Elección Pública, se caracteriza por integrar un conjunto de conceptos y técnicas, no siempre dotado de la coherencia apropiada, susceptibles en principio de mejorar la eficacia y eficiencia de las Administraciones Públicas. En el plano de las presunciones con frecuencia no suficientemente explicitadas, cabe decir dos cosas, en primer lugar, que la NGP parte de una visión superior del mercado respecto de la gestión pública tradicional, aunque no llega a forzarla hasta el extremo de no reconocer las particularidades del sector público, y, en segundo, que reco- 
noce un concepto distinto de la «gestión» frente a la mera «administración» (Olías de Lima, 2002). En torno a esto último, debe reseñarse que, en efecto, la gestión pública implica la movilización de todo tipo de recursos, básicamente en el sector operativo, pero sin excluir la participación interna y externa en la determinación de fines y objetivos, apostando por dar una mayor discrecionalidad en la elección de los recursos y procedimientos, por la posibilidad de obtener recursos recurriendo a la financiación externa y haciendo hincapié, como no podía ser de otra manera, en la evaluación y asignación de responsabilidades, así como en el capital humano.

En ese marco ofrece un conjunto de propuestas que impactan sobre los elementos nodales de la Administración, destacando las que afectan a las estructuras (se enfatiza la coordinación gubernamental y, en el plano netamente administrativo, aspectos como la reducción del tamaño del aparato público, la creación de agencias y el aplanamiento de las jerarquías), a los presupuestos (preconizando la presupuestación y gestión por objetivos), y al singular mercado público, apostando por la introducción de mecanismos cuasicompetitivos y de mercado que operan tanto sobre la oferta (a través, por ejemplo, de la contratación externa, privatizaciones, la construcción de mercados internos, el benchmarking...) como sobre la demanda (mediante, v.g., vales públicos, precios públicos, tasas) (Albi, 1997; López, 2003). Y ello sin olvidar las previsiones en torno a las preferencias de los ciudadanos/clientes, que se constituyen pretendidamente en el eje de la acción pública, dando lugar a una preocupación constante tanto de la mejora de los servicios públicos como de la comunicación con los beneficiarios a través de diversas técnicas, entre las que destacan la Administración electrónica o la aplicación de la calidad (Ballart, 2001; Bazaga et alia, 1998; Crespo González, 2001; Criado, 2004; Gómez Rivas, 2004; López y Gadea, 2001; Sancho, 1999).

Especialmente relacionada con la modernización administrativa de las Administraciones españolas se encuentra la introducción de la calidad, concepto en que subyacen dos ideas esenciales, la de que se trata de un proceso continuo que integra la adaptación y el cambio, y la que indica que el cambio y la mejora permanentes están asociadas a un profundo proceso de comunicación y participación, dentro y fuera de la organización. En este sentido, entre las distintas herramientas e instrumentos que se asocian a la calidad en la Administración cabe reseñar las que son objeto de este trabajo: las Cartas de Servicios (CdS) ${ }^{5}$ (Pollit y Harrison, 1992).

${ }^{5}$ Otras herramientas vinculadas frecuentemente a la introducción de la calidad, además de las citadas CdS, son: la introducción del sistema de planificación y programación por objetivos, con indi- 
Las CdS, que inicialmente nacieron en Reino Unido como un producto de las políticas thacheristas de reforma del sector público ${ }^{6}$ con objeto de transformar radicalmente los servicios, enfatizando los resultados y la satisfacción del ciudadano/cliente, y acercándolos a la esfera del sector privado, han hecho fortuna en los distintos países de la OCDE, aunque bajo formulaciones y orientaciones diversas (McGuire, 2001; Olías de Lima, 2003; Pollit, 1994).

En España, la Comunidad de Madrid reconoció pronto ${ }^{7}$ el potencial de este instrumento para fomentar la mejora continua de los servicios públicos y explicitar los niveles o estándares de calidad con los que los ciudadanos pueden esperar que se presten los servicios (DGCS, 1998; Moreno, 1998). De esta forma, mediante el Decreto 27/1997, de 6 de marzo, reguló las CdS, los sistemas de evaluación de la calidad y los premios anuales a la excelencia y calidad del servicio público. Más tarde, aprobó medidas complementarias, como el establecimiento del contenido mínimo (Decreto 44/1998, de 18 de marzo) con que debían contar, sin prejuzgar la posibilidad de elaborar cartas con un contenido más amplio, particularmente incluyendo datos sobre estándares de calidad e indicadores.

De esta manera, la Administración de la Comunidad de Madrid ha asumido «darse a conocer al ciudadano a través de las Cartas de Servicios, mediante la divulgación de los servicios, todo ello a fin de promover la mejora continua de los servicios que presta al ciudadano, debido a que con frecuencia los ciudadanos desconocen el potencial de los servicios que la Administración ofrece y de los cuales pueden beneficiarse ${ }^{8}$.

cadores de calidad; los sistemas de reclamaciones y quejas de los ciudadanos; los estudios de calidad de los servicios y satisfacción de los usuarios; grupos de mejora y sistemas de sugerencias de los empleados; y la autoevaluación del sistema introducido a través del modelo EFQM.

${ }^{6}$ La primera carta fue aprobada a finales de 1991, a iniciativa de John Major.

${ }^{7}$ En todo caso antes que la Administración del Estado, que introdujo con carácter general las CdS en 1999, mediante el R.D. 1259, de 16 de julio. El proceso de introducción de esta herramienta de calidad ha sido cuantitativamente exitoso, pues desde entonces son centenares los servicios de todas las Administraciones españolas que han elaborado y publicado sus propias $\mathrm{CdS}$, aunque los resultados de su introducción sean muy diversos y desiguales, lo que impide generalizar.

8 Tomado de http://madrid.org/pres_atención_ciudadano/calidad_servicios/cartas_servicios (fecha de acceso: 6 de mayo de 2004). 


\section{CONTENIDO MÍNIMO Y AMPLIO DE LAS CARTAS DE SERVICIO DE LA COMUNIDAD DE MADRID}

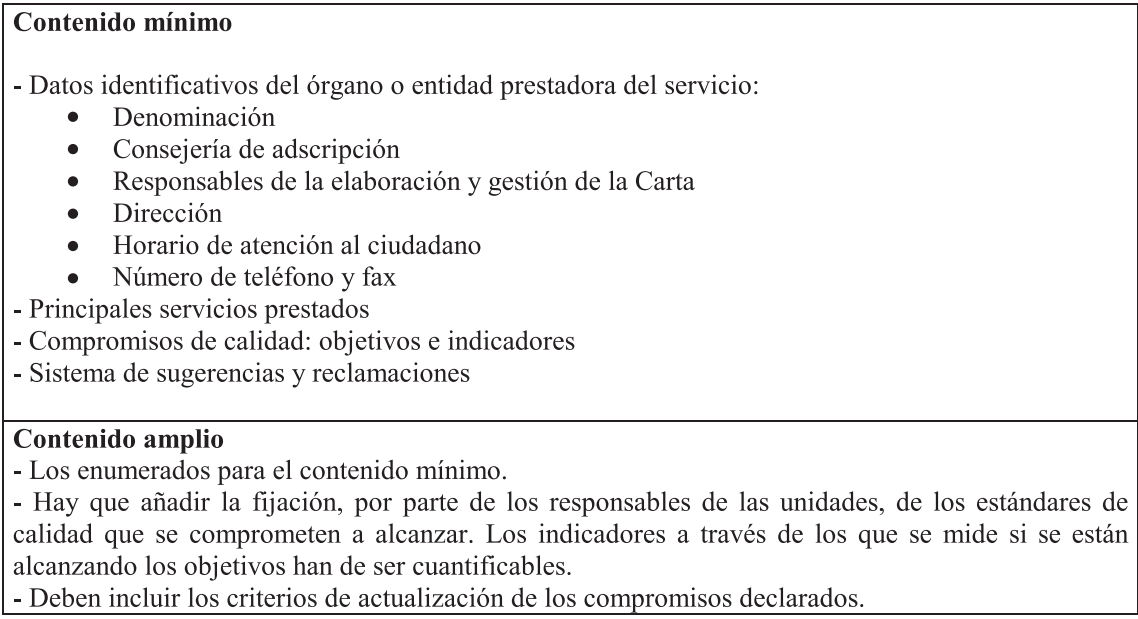

Fuente: Decreto 27/1997, de 6 de marzo, y Decreto 44/1998, de 18 de marzo, de la Comunidad de Madrid

Los autores estiman que las CdS son un buen referente para conocer en qué medida se ha producido una mejora de los servicios en la CM (perspectiva intra-organizativa y extra-organizativa), a partir del inicio de la introducción de aspectos vinculados a la NGP, y particularmente de la calidad y de las CdS. A ello coadyuvan los siguientes factores:

a) La importancia que en la elaboración y actualización de las CdS se otorga a aspectos tales como los estándares de calidad, eficacia, eficiencia, accesibilidad y buen trato, así como en la necesidad de escuchar al ciudadano a través de sus quejas y reclamaciones.

b) Los ámbitos políticos y organizativos en que impactan las CdS son muy relevantes socialmente, yendo desde los relacionados con la legitimidad democrática (institucional y por resultados) a aquellos que enfatizan los derechos de los ciudadanos y sus garantías en el contenido de los servicios, sin olvidar los que afectan a la mejora de los servicios y a la responsabilidad de los gestores, promoviendo sistemas de evaluación. De esta manera, las CdS ofrecen la posibilidad de mejorar el control y la participación ciudadana tanto sobre los actos y productos públicos, como sobre las decisiones atinentes a su configuración, implantación y evaluación.

c) Desde una perspectiva de análisis intra-organizativa, Las CdS desprenden potencialidades para la mejora de los servicios, lo que afec- 
taría a todos los factores administrativos: diseño orgánico, recursos humanos, recursos materiales y tecnológicos, modos de gestión y sistemas de información y comunicación.

Como es sabido, conocer hasta qué punto esto último es cierto es el objeto de nuestro estudio, y para ello, por su importancia e interés, nos centramos en dos factores esenciales: los recursos humanos y los recursos materiales y tecnológicos. La importancia de dichos factores para la gestión pública y la mejora de los servicios es evidente y no serían necesarios más datos, pero creemos de interés destacar, en el plano de introducción de la calidad y de la NGP en que aparecen las CdS, que forman parte de los nueve criterios del modelo EFQM de Excelencia 2000, tanto en el grupo de los agentes facilitadores como en el de resultados, y que, además, son esenciales para entender cuáles son las propuestas de valor y satisfacción que ofertan los servicios, y, en su caso, las diferencias (gaps) existentes con las valoraciones percibidas por los beneficiarios de los productos y/o servicios.

\section{RESULTADOS SOBRE EL CAPITAL HUMANO}

Como es conocido, las personas o el capital humano al servicio de las Administraciones no constituyen un factor organizativo más, sino el más importante: entre otras cosas, su concurso es imprescindible para concretar e implantar los fines y objetivos intra y extra-administrativos. Tanto esto es así, que si trasladamos la afirmación a nuestro objeto de investigación podríamos concluir que el análisis del impacto sobre el personal de la producción y gestión interna de las CdS es un buen indicador para entender en qué medida su introducción en la CAM ha producido cambios organizativos, pues si éstos se hubieran producido con cierta intensidad sin duda habrían de reflejarse en las actividades que conforman la gestión del personal.

Es por ello que en este epígrafe nos planteamos dos preguntas de relieve para conocer el devenir intra-organizativo de las CdS:

1.- ¿Cómo se ha producido la participación de los distintos tipos de personal en el diseño, implantación y evaluación de las cartas?

2.- ¿Qué influencia ha tenido la introducción de las cartas sobre las actividades de gestión del personal?

Con la primera pregunta queremos vislumbrar en qué medida los distintos colectivos del personal han participado en los aspectos relativos a 
la creación, implantación y evaluación de los aspectos recogidos en las $\mathrm{CdS}$, mientras que con la segunda, intentamos aproximarnos al posible impacto que la introducción de las mismas han podido tener en actividades relevantes (acceso, formación, promoción, retribuciones, diseño y modificación del contenido de los puestos, motivación, satisfacción y actividades de reconocimiento) vinculadas a la gestión y administración de personal en la Administración de la CAM. Con tal fin, hemos utilizado como fuentes tanto la información aportada por los 44 servicios que respondieron al cuestionario como la información obtenida por los miembros de esta investigación mediante las entrevistas en profundidad efectuadas (dicha información se ha revelado como de gran utilidad para contrastar la proporcionada por los cuestionarios, la cual podría haber sido no infrecuentemente remitida en términos autojustificativos e incluso buscando lo socialmente, o mejor, lo «político-administrativamente» aceptable).

\subsection{La participación de los colectivos de personal en la formulación, implantación y evaluación de las Cartas de Servicios}

Habiendo excluido voluntariamente al personal político, cuyo respaldo a unas directrices generales ha podido ser imprescindible para la introducción de los procesos de modernización y calidad en la CAM, y singularmente para la concreción de la idea de las CdS, y pensando que dicho personal no se habría implicado directamente en aspectos operativos o prácticos en lo concerniente a la construcción, implantación y evaluación de las cartas en los servicios individualmente considerados, hemos optado por centrarnos en señalar exclusivamente la implicación del personal de cada unidad con carta, diferenciando entre directivos, personal operativo y representantes del personal.

a) El Personal Directivo. El personal directivo es el que más se ha implicado en los distintos aspectos analizados en torno a la CdS, de forma que los cuestionarios expresan una fuerte participación en la formulación y diseño (cerca del 91\% lo ha hecho mucho o bastante) y en la implantación de la misma (más del $86 \%$ lo ha hecho mucho o bastante). Su actividad en la evaluación de los resultados asociados a la CdS ha sido algo menor, aunque también importante (el $61,37 \%$ lo ha hecho mucho o bastante).

Los datos anteriores son bastantes coherentes, sobre todo los que aluden al diseño, con lo observado en otras preguntas, como la que interroga 
sobre el origen de la introducción de la carta en su unidad, en que se constata que la idea suele venir de los directivos o técnicos que han recibido formación sobre calidad, o a los que se les trasladó en su momento la necesidad de su elaboración por la Dirección General de Calidad de los Servicios de la CAM. Esto también se ve refrendado por las entrevistas en profundidad.

La visión sin embargo dista de ser tan unívoca cuando se interroga en torno a si el personal directivo toma como criterio de actuación el contenido de la CdS de su unidad. Si bien el 66\% dice hacerlo mucho o bastante, en las entrevistas no son infrecuentes los comentarios del tipo: «los directivos y los técnicos de la unidad no toman en consideración de manera consciente la $\mathrm{CdS}$ en sus actuaciones, más bien se rigen por los determinantes normativos que afectan a su actividad» ${ }^{9}$, aseveración que también será de aplicación al personal operativo.

Por otra parte, cabe destacar que los directivos no han recibido en general formación sobre las CdS (sólo un 36,36\% la ha recibido contra el $52,27 \%$ que no), y que con frecuencia la formación no ha sido monográfica sobre las mismas sino que se encontraba inserta dentro de un curso sobre calidad realizado. En todo caso, sí ha habido dentro de este colectivo intención de compartir sus conocimientos sobre $\mathrm{CdS}$ con el resto del personal de su unidad, ya que casi el $70 \%$ reconoce haberlo hecho mucho o bastante, frente al $12,50 \%$ que lo ha hecho poco. Con todo, cabe reseñar que, en términos generales, la introducción de las CdS no se ha visto acompañada con un plan de comunicación específico.

b) El Personal Operativo. Este tipo de personal, de acuerdo con los cuestionarios, habría tenido una implicación relativamente importante en la formulación y diseño de la Carta (66\% mucho o bastante), en la implantación de la misma $(63,64 \%$ mucho o bastante) y destacable en la evaluación de sus resultados (45,46\% mucho o bastante).

En este caso, las informaciones obtenidas a través de los cuestionarios no se comparecen con la obtenida mediante las entrevistas. En éstas, aparte de reconocer que lo que principalmente se tenía en cuenta eran los determinantes normativos que afectaban a su actividad, fueron muy frecuentes expresiones como las siguientes: «los funcionarios no se involucran, "pasan". Ven las cartas con indiferencia o recelo» ${ }^{10}$, «...Digo como confidencia que la $\mathrm{CdS}$ resulta desconocida para el propio personal del

\footnotetext{
9 Entrevista al Servicio de información y documentación ambiental.

10 Entrevista a las Instalaciones Deportivas del IMDER.
} 
organismo, ni siquiera los directores de unidades conocen su existencia....» ${ }^{11}, \ll \ldots$... el personal por parte del director del laboratorio, sin embargo, muchas de esas personas ni siquiera han leído su contenido...o, directamente, no les interesa o rechazan este tipo de herramientas» ${ }^{12}$.

De esta forma, y de acuerdo con las entrevistas, parece posible afirmar que la implicación del personal es mayor en la medida en que aumenta el componente técnico en la unidad, y el número de empleados no es muy alto. En ocasiones también se producen reuniones periódicas para tratar con los trabajadores la marcha del servicio y el cumplimiento de los aspectos recogidos en la Carta o, incluso, para hablar de su eventual reforma y/o actualización (sobre todo en lo referido a indicadores).

c) Los representantes del personal. Los representantes del personal han tenido un papel muy reducido en la introducción y evaluación de la CdS. En la formulación y diseño de la carta, así como en la evaluación de los resultados, sólo un exiguo $16 \%$ de los cuestionarios afirma mucha o bastante participación (contra el $66 \%$ que en ambos casos informa de un poco o nada participación); mientras que el $15,01 \%$ de los cuestionarios reconocería mucha o bastante implicación en la implantación de la CdS, frente al casi $66 \%$ que indicaría poco o nula participación. En las entrevistas también se encontraron datos que confirman esa escasa o casi nula participación; como exponente de todos sirva la siguiente afirmación: «El papel de los representantes de personal ha sido ninguno y de haber intervenido habría sido para pedir dinero, y no en la parte técnica» ${ }^{13}$.

Una de las conclusiones más evidentes de nuestro estudio es la práctica ausencia de impacto desde la perspectiva de gestión interna y del personal como consecuencia de la introducción de las CdS. Pues bien, con independencia de que la escasa o nula participación de los representantes del personal sea causa o consecuencia de esa conclusión, o acaso ambas cosas a la vez, puede decirse que estamos ante un aspecto de gran interés y que puede realizar funciones de contraste del discurso modernizador sobre las CdS y su eficacia real. En efecto, de tener consecuencias concretas dicho discurso las mismas se proyectarían sobre el empleo público de las unidades con carta (aunque bajo diferentes formas e incidencia) y ello conllevaría, de acuerdo con la legislación vigente, una participación

\footnotetext{
11 Entrevista al Centro de Transfusión de la Comunidad de Madrid.

12 Entrevista al Laboratorio Regional de Salud Pública.

13 Entrevista al Consorcio Regional de Transportes.
} 
obligada y una negociación preceptiva entre las autoridades administrativas y los representantes del personal. Y ello, sin duda, significaría una implicación cualitativa y significativa de los representantes del personal en los procesos de formulación, implantación y evaluación de la CdS de su unidad. A esa conclusión nos conduce la Ley 9/1987, de 12 de junio, de órganos de representación, determinación de las condiciones de trabajo y participación del personal al servicio de las Administraciones Públicas (BOE de 17 de julio de 1987), sobre todo en lo explicitado en sus artículos 32 y $34^{14}$.

En el caso que nos ocupa, es claro que si la introducción de la CdS realmente afectara a las actividades de gestión del personal reconocidas como objeto de negociación de acuerdo con el artículo 32 citado, la implicación de los representantes del personal sería alta, pues deberían negociar con carácter preceptivo, por más que en caso de que no se produzca acuerdo, o no se produzca una aprobación expresa y formal, correspondería a las autoridades administrativas establecer las condiciones de trabajo de los

\footnotetext{
14 Téngase en cuenta que de la legislación que a continuación se expone ha quedado excluido «el personal laboral al servicio de las distintas Administraciones Públicas, que se regirá por la legislación laboral común, sin perjuicio de lo previsto en los artículos 39 y 40 y en la disposición $5^{\mathrm{a}}$ » (artículo 2.1.d, de la Ley 9/1987, de 12 de mayo). En lo que nos interesa, los citados preceptos se refieren a la composición del Consejo Superior de la Función Pública, y a la facultad de las Administraciones Públicas para establecer órganos colegiados para la participación de las organizaciones sindicales en las materias relacionadas con el sistema retributivo y el régimen del personal a su servicio.
}

Artículo 32: Serán objeto de negociación en su ámbito respectivo y en relación con las competencias de cada Administración Pública las materias siguientes: El incremento retributivo de los funcionarios y del personal estatutario...La determinación y aplicación de las retribuciones de los funcionarios públicos. La preparación y diseño de los planes de oferta de empleo público. La clasificación de los puestos de trabajo. La determinación de los programas y fondos para la acción de promoción interna, formación y perfeccionamiento. La determinación de las prestaciones y pensiones de las clases pasivas y, en general, todas aquellas que afecten de algún modo, a la mejora de las condiciones de vida de los funcionarios jubilados. Los sistemas de ingreso, provisión y promoción profesional de los funcionarios públicos. Las propuestas sobre derechos sindicales y de participación. Medidas de salud laboral. Todas aquellas que afecten, de algún modo, al acceso a la función pública, carrera administrativa, retribuciones y seguridad social, o a las condiciones de trabajo de los funcionarios públicos y cuya regulación exija norma con rango de Ley. Las materias de índole económica, de prestación de servicios sindical, asistencial, y en general cuantas otras afecten a las condiciones de trabajo y al ámbito de relaciones entre funcionarios públicos y sus Organizaciones Sindicales con la Administración.

Art. 34. 1. Quedan excluidas de la obligatoriedad de la negociación, en su caso, las decisiones de las Administraciones Públicas que afecten a las potestades de organización, al ejercicio de los derechos de los ciudadanos ante los funcionarios públicos y al procedimiento de formación de los actos y disposiciones administrativos. 2.- Cuando las consecuencias de las decisiones de las Administraciones Públicas que afecten a sus potestades de organización puedan tener repercusión sobre las condiciones de trabajo de los funcionarios públicos, procederá la consulta a las Organizaciones Sindicales y Sindicatos a que hacen referencia los artículos 30 y 31.2 de la presente Ley. 
funcionarios públicos (artículo 37). Estas extensas apreciaciones son necesarias para comprender los resultados que se presentan en las líneas siguientes.

\subsection{La influencia de la introducción de las Cartas de Servicios sobre las actividades de gestión de personal}

El examen de los datos recogidos en los cuestionarios, junto a los obtenidos a partir de las entrevistas, muestran una escasa incidencia en las actividades de gestión de personal, lo que da cuenta del exiguo impacto que en términos de gestión interna han tenido las CdS. Para fundamentar la afirmación precedente nos centraremos en su influencia sobre los aspectos vinculados a la gestión de personal que se presentan a continuación.

a) El acceso a los puestos de la unidad. En términos generales puede decirse que sólo de manera marginal se tienen en cuenta los aspectos recogidos en la CdS para el acceso a los puestos (el 18,18\% expresa que se tiene en cuenta bastante, mientras que el $63,63 \%$ de los cuestionarios estima que poco o nada). En algunas entrevistas se reconoció que si bien la introducción de la carta no había provocado cambios sustantivos en la gestión de los servicios, ni en los recursos humanos, ni en el diseño de los puestos, sí podía decirse que había evidenciado la necesidad de «cuidar los empleados que prestan servicios de atención al ciudadano» ${ }^{15}$.

b) La formación de los empleados de la unidad. Es éste un aspecto vinculado a la gestión de personal en que sí parece haberse producido una cierta incidencia por la adopción de la CdS. De esta forma, un $45,45 \%$ de las respuestas obtenidas en los cuestionarios reconocen que dicha introducción ha influido mucho o bastante en la formación de los empleados, en tanto que el $40,91 \%$ estimaba que poco o nada. Además de lo escrito más arriba sobre la formación del personal directivo y en qué medida dicho colectivo compartía sus conocimientos sobre las $\mathrm{CdS}$ con el resto del personal, las entrevistas muestran que en general se solicita una formación más específica sobre el particular, pues la mayoría de la recibida se asocia a cursos genéricos sobre calidad en que se insertarían aspectos generales sobre las Cartas y no tanto acciones formativas monográficas sobre las CdS, en que aparte de las inevitables aportaciones conceptuales se ofreciera información y destrezas, entre otras cosas, para definir los

15 Entrevista al Consorcio Regional de Transportes. 
indicadores del servicio, auténtico tendón de Aquiles de la mayoría de ellas. De esta manera se podría salir al paso de afirmaciones como «no se imparte una formación específica a los funcionarios relacionada con las Cartas ${ }^{16}$, o que «La formación recibida sobre CdS por el personal ha sido mínima» ${ }^{17}$.

c) La promoción del personal de la unidad. La promoción del personal de la unidad no se vincula con la CdS. Sólo un porcentaje cercano al 7\% de las respuestas de los cuestionarios estima mucha o bastante incidencia de la misma en ese proceso, mientras que el porcentaje que indica poco o ninguna influencia asciende al $75 \%$. Lo que fue validado por las entrevistas, donde no era infrecuente escuchar que «la $\mathrm{CdS}$ no influye en procesos internos ni en la promoción del personal» ${ }^{18}$. Estos resultados están relacionados con tres hechos: el primero es que las actividades de promoción están sujetas a una regulación muy estricta, tanto del proceso como de las características de los méritos a aportar; el segundo, es que, como ocurre para otros aspectos, no es fácil diferenciar y valorar el desempeño de los empleados individuales respecto a la carta; y, finalmente, encontramos que el modelo informalmente establecido de reconocimiento de los servicios prestados en torno a lo descrito por la carta, está fundado en general en meras declaraciones de gratitud.

d) La retribución de los empleados de la unidad. Lo escrito anteriormente también es válido para comprender el nulo impacto de las $\mathrm{CdS}$ sobre las retribuciones del personal. En efecto, sólo el 2,27\% de las respuestas de los encuestados explicitaban que la introducción de la carta en su unidad había incidido mucho o bastante en los salarios, en tanto que el $80 \%$ opina que la influencia ha sido nula o poca. Estos datos, tajantes y significativos, han sido confirmados por las entrevistas y, junto con casi todos los aportados anterior y posteriormente respecto de otras actividades de gestión de personal, están en consonancia con la poca participación de los representantes del personal en todo lo relativo a las cartas, como ya se puso de manifiesto anteriormente.

e) La modificación del contenido de los puestos de la unidad. Las CdS han tenido una incidencia tangencial en otro ámbito, el que alude a la modificación del contenido de los puestos con motivo de su introducción. Sólo un exiguo 16\% de las respuestas al cuestionario reconocen mucha o

\footnotetext{
16 Entrevista a las Instalaciones Deportivas del IMDER.

17 Entrevista al Laboratorio Regional de Salud Pública.

18 Entrevista al Conservatorio profesional de música de Amaniel.
} 
bastante incidencia en esa actividad, mientras que el $66 \%$ estima lo contrario, una poco o nula influencia. Esa escasa influencia ha sido confirmada en las entrevistas, si bien alguna vez se expresó que con la adopción de la CdS se había evidenciado la necesidad de personal informático ${ }^{19} \mathrm{y}$ de una preocupación por los puestos de atención a los ciudadanos ${ }^{20}$.

f) La motivación y satisfacción de los empleados de la unidad. La CdS parece haber incidido mucho o bastante en la motivación del personal para el $27,28 \%$ de las respuestas al cuestionario, en tanto que poco o nada para el $61,36 \%$, siendo idénticos los datos en lo relativo a si la Carta de Servicios ha mejorado la satisfacción laboral del personal de la unidad. Los datos de entrevistas no aportan grandes novedades. Si acaso, señalar que en algunas aparece la duda de si la motivación y satisfacción del personal en realidad sólo afecta al personal de dirección encargado de la Carta, ya que el resto con gran frecuencia simplemente conoce su existencia y, en caso de estar motivado, no lo haría tanto por ésta como por el contenido de su trabajo ${ }^{21}$.

g) El sistema de reconocimiento del personal. De acuerdo con los datos de los cuestionarios, lo frecuente es que no exista un sistema de reconocimiento dirigido a los empleados de la unidad por el trabajo bien hecho en relación con la CdS (Sólo el 15,91\% dice disponer de uno, frente al $72,73 \%$ que dice no poseerlo). Si se afina un poco más el análisis, y siempre según los datos de los cuestionarios, las pocas unidades que tienen un sistema de reconocimiento en torno a la CdS lo articulan esencialmente sobre el reconocimiento informal, ya sea público o privado $(75,43 \%)$ y, en mucha menor medida, sobre otros indicadores. Las entrevistas, por su parte, no han conseguido verificar la existencia de tales sistemas de reconocimiento, seguramente por el carácter marcadamente informal (no institucionalizado) que se ha destacado en los cuestionarios.

\section{RESULTADOS SOBRE LOS RECURSOS MATERIALES Y TECNOLÓGICOS}

Los recursos materiales y tecnológicos forman parte del sistema nervioso central y componen el armazón básico de las organizaciones admi-

\footnotetext{
19 Por ejemplo, en la entrevista al Servicio de información y documentación ambiental.

${ }^{20}$ Por ejemplo, en la entrevista al Consorcio Regional de Transportes.

21 Información en ese sentido viene recogida, por ejemplo, en la entrevista al Conservatorio profesional de música Amaniel.
} 
nistrativas. El análisis de los recursos vinculados con las CdS cuenta con una importancia más que notable en el presente estudio. En este apartado se pretendía profundizar en la incidencia de las CdS en la adopción y difusión de nuevos recursos materiales y tecnológicos en las unidades y servicios objeto de estudio, ya que si las CdS responden realmente a una visión diferente del servicio a los ciudadanos es necesario una revisión de los procesos y los objetivos de las organizaciones públicas, estableciendo instrumentos de mejora y sistemas eficaces de control y responsabilidad por los resultados, lo cual no es posible sin una adecuación de los recursos disponibles.

Los resultados obtenidos se sitúan en la línea de lo que se ha ido planteando a lo largo de este trabajo. Se confirma la atención limitada que las diferentes unidades administrativas han dispensado a las $\mathrm{CdS}$, teniendo en cuenta la incorporación de nuevos recursos y, por tanto, la relativa incidencia de estas herramientas como correas de transmisión del cambio en la gestión pública autonómica. Sin embargo, no deja de ser cierto que se pueden encontrar determinadas variaciones entre las diferentes categorías abordadas, lo que permite calibrar mejor qué tipo de recursos son los que se han asociado más a las CdS. En concreto, parece que el discurso asociado al cambio tecnológico parece que ha tenido incidencia en la Administración autonómica, aunque no está del todo claro que ello suponga verdaderos resultados de cara a la difusión de innovaciones en la gestión pública.

\subsection{Futuras dotaciones presupuestarias en torno a las Cartas de Servicios}

Como cuestión preliminar, se planteó la previsión en un futuro inmediato de un incremento presupuestario vinculado a la implantación de los aspectos recogidos en las CdS. El hecho de que las diferentes dimensiones vinculadas a la implementación de las CdS no lleve aneja una previsión generalizada de incremento presupuestario en las unidades dentro de las que se han aprobado indica su limitado alcance. Las entrevistas en profundidad realizadas confirmaron que no existe la perspectiva de aumentar esas dotaciones presupuestarias y, de hecho, no se han planteado necesidades específicas, ni siquiera en determinados sectores de la Administración autonómica donde podían ser más previsibles, es decir, aquellos con un impacto más directo sobre la atención ciudadana y la prestación de servicios finalistas.

En términos cuantitativos, las respuestas al cuestionario confirmaron la existencia de una amplia mayoría de unidades administrativas que no con- 
templan en el futuro próximo un aumento del presupuesto que se pueda asociar directamente a las CdS (52,7\%). No obstante, se puede introducir un pequeño matiz a esta visión general. Por una parte, la tasa de no respuesta resulta significativamente elevada en esta pregunta $(31,82 \%)$ y, por otra parte, también se ha descubierto una pequeña cantidad de organismos que responden afirmativamente a esta cuestión, es decir, que sí prevén algún tipo de nuevo gasto $(15,91 \%)$. En este sentido, aunque no se dispone de mayor detalle sobre el alcance de esos futuros incrementos en la financiación asociada a las CdS, sí se pueden conseguir algunas evidencias teniendo en cuenta lo que se ha producido en el pasado reciente en torno a los diferentes tipos de recursos disponibles por las organizaciones administrativas para llevar a cabo su labor.

En concreto, se puede subrayar una doble corriente relacionada con el presupuesto vinculado a las CdS. Por un lado, la unidad central encargada de su promoción en el conjunto de la organización, la Dirección General de Calidad de los Servicios y Atención al Ciudadano, ha realizado un considerable esfuerzo por nutrir de contenido económico a este tipo de iniciativa, de manera que financia de forma generosa diferentes aspectos necesarios para su implantación. Por otro lado, a nivel operativo los diferentes servicios y organismos en los que se han implantado las $\mathrm{CdS}$ no han previsto o conseguido de manera generalizada incrementos presupuestarios notables asociados a la materia, de manera que en muchos casos no se considera el potencial de estas herramientas para conseguir y obtener resultados importantes derivados de la implantación. En definitiva, el compromiso presupuestario que se precisa para concretar cambios de envergadura no se ha realizado de la manera que hubiera sido deseable de cara a consolidar experiencias de cambio dentro de la Administración autonómica.

\subsection{La mejora de los recursos humanos, materiales y tecnológicos}

En este apartado interesaba conocer con más profundidad los tipos de recursos que se han asociado a la implantación de las CdS. Aquí se diferenciaron varias categorías (recursos humanos, recursos tecnológicos, inversiones materiales y otros), de cara a disponer de una idea más nítida sobre las implicaciones económicas de este tipo de herramientas en las unidades administrativas en las que se han adoptado. En consonancia con la ausencia de previsiones de nueva financiación en el futuro, también se han observado limitaciones en las aportaciones humanas, tecnológicas y de inversiones materiales en relación con las $\mathrm{CdS}$ en el pasado, tal y como 
se encargaron de corroborar muchos de los entrevistados en diferentes unidades administrativas ${ }^{22}$. De hecho, esto no es más que la traducción de las carencias contrastadas en las dimensiones previas de análisis, especialmente, en el plano de los recursos humanos.

El cuestionario puso de manifiesto determinadas diferencias entre las categorías analizadas. Tanto los recursos humanos $(15,91 \%)$ como las inversiones materiales $(22,73 \%)$ concentraron el menor nivel de respuestas entre las opciones mucho y bastante, en referencia a la utilización de nuevos recursos asociados a la implantación de las CdS. La dimensión tecnológica parece que se ha contemplado en mayor medida como necesaria, dado que casi un $30 \%$ de las unidades analizadas confirma incrementos considerables de recursos en esta esfera. En todo caso, los resultados son más bien discretos en todas las categorías, incluyendo la opción abierta otros, que no fue seleccionada en ninguno de los cuestionarios recibidos.

Estos datos sugieren la idea de las $\mathrm{CdS}$ como instrumentos que han permitido justificar determinados gastos extraordinarios, fundamentalmente, en el plano tecnológico, mientras las dimensiones humana y material han quedado prácticamente relegadas de esos aumentos. Parece que las $\mathrm{CdS}$, como otras prácticas y estrategias relacionadas con la modernización administrativa, se han revestido del discurso tecnológico como una manera de aliviar las necesidades informáticas de las organizaciones, asociando su implantación a nuevas inversiones en el ámbito telemático. Además, con ello se ha tratado de justificar determinadas acciones de reforma que no cuentan con un claro encaje en algunas unidades o servicios con culturas administrativas altamente burocráticas. Sin embargo, resulta interesante profundizar un poco más en este ámbito de cara a contrastar el alcance de estas conclusiones.

\subsection{Las tecnologías en las Cartas de Servicios}

El interés específico por los recursos tecnológicos llevó a plantear de manera más concreta su relación con las $\mathrm{CdS}$. Las entrevistas mostraron que algunas unidades administrativas han percibido las innovaciones tecnológicas e informáticas como un medio con cierto potencial para cola-

\footnotetext{
${ }^{22}$ Cabe mencionar que casi todos los organismos analizados destacaron la financiación por parte de la Dirección General de Calidad de los Servicios y Atención al Ciudadano de los dípticos que informan sobre el contenido de las Cartas de Servicios.
} 
borar en la efectiva implementación de las CdS. En este sentido, también se puso de manifiesto que las CdS, al menos de forma mínima, requieren una combinación con nuevas aplicaciones informáticas o tecnológicas, bien para explorar su dimensión de atención ciudadana (por ej., mediante su difusión vía web), bien para mejorar la dimensión de gestión interna (por ej., a través del tratamiento de indicadores y la creación de cuadros de mando para el apoyo a las decisiones).

Los cuestionarios reflejaron la orientación de las cuestiones mencionadas. En más de un 36\% de las unidades administrativas analizadas se ha considerado que la implantación de las CdS ha conllevado la incorporación de nuevas herramientas tecnológicas. Este dato confirma la visión creciente en torno al discurso tecnológico, que a veces tiende a relacionar de manera inadecuada las innovaciones tecnológicas y la modernización de la gestión pública, sin una reflexión previa sobre las propias necesidades organizativas o las potencialidades de las tecnologías, especialmente, dentro de determinados entornos institucionales con importantes resistencias a su utilización efectiva.

De cara a ahondar en la interacción apuntada, se planteó de manera abierta cuáles eran esas nuevas aplicaciones informáticas utilizadas y para qué se habían adoptado. Aunque el número de respuestas disponibles no permite plantear los resultados como generalizables, al menos apuntan algunas pistas sobre la dirección de esta relación. Por un lado, las nuevas herramientas se refieren fundamentalmente a programas y soportes informáticos, así como sistemas de información, aunque en menor medida. Por otro lado, el objeto de la incorporación se ha unido mayoritariamente a la comunicación interna, el mantenimiento y análisis de información, la promoción de proyectos y la agilización de la gestión. Como se ha adelantado, esto confirma una perspectiva todavía limitada respecto al potencial de las tecnologías en la mejora de la gestión pública, aunque confirma que se trata del tipo de recursos más utilizado por los organismos objeto de estudio para consolidar las $\mathrm{CdS}$.

\section{REFLEXIONES SOBRE LAS CARTAS DE SERVICIOS Y SUS RESULTADOS}

Las CdS son una de las herramientas de mejora de la calidad más adoptada por las Administraciones públicas españolas, sin embargo, resulta necesario calibrar los resultados de su adopción, así como las consecuencia que ello supone para el estudio de las reformas administrativas en marcha. El análisis de las dos secciones anteriores se completa con unas refle- 
xiones sobre los temas ya tratados, así como una recapitulación sobre las CdS y su papel dentro de la calidad y su aplicación específica a la gestión pública. Los datos disponibles sobre las CdS han demostrado diferentes aspectos de las cuestiones abordadas, si bien se pretende hacer un balance teniendo en cuenta las entrevistas en profundidad y las numerosas apreciaciones realizadas por las personas que han participado en la investigación dentro de las diferentes unidades administrativas seleccionadas y las respuestas a dos preguntas abiertas del cuestionario, que fueron respondidas en la mayoría de los casos, aunque con diferentes niveles de detalle. Teniendo en cuenta todo lo anterior, finalmente se retoman los argumentos expresados inicialmente sobre las CdS y su papel en la gestión de las Administraciones públicas, de manera que se intenta arrojar luz al debate dentro de la disciplina sobre la implantación de la calidad en el sector público y sus repercusiones para la gestión pública.

\subsection{Balance específico: las Cartas de Servicios y los recursos disponibles}

Las CdS han supuesto un reto para las organizaciones en las que se han adoptado, tanto para el personal a su servicio, como en lo referente a los recursos materiales y tecnológicos disponibles. De manera genérica, se puede afirmar que la puesta en marcha de las CdS en los organismos objeto de estudio ha supuesto una serie de dificultades, sobre todo, problemas relacionados con las características institucionales de las unidades administrativas, tales como el presupuesto, el personal, el cambio organizativo, la normativa, etc. Por otra parte, en lo que respecta a las principales aportaciones de las CdS hay que decir que se han orientado en torno a determinados aspectos como la divulgación del servicio o unidad en el exterior, la mejora genérica del servicio o del compromiso con los ciudadanos, así como cuestiones internas relacionadas con la identificación de puntos débiles, el logro de una mayor implicación del personal en la actividad corriente o la adquisición de nuevas herramientas tecnológicas. Si bien las CdS no han servido de motor para promover cambios notables en la gestión, al menos han funcionado como difusores de otro tipo de transformaciones de mayor alcance, convirtiéndose a veces en herramientas de comunicación limitadas en cuanto a sus resultados.

El problema del capital humano es el principal en relación al alcance de las CdS. La investigación ha puesto de manifiesto la falta de implicación del personal de las unidades analizadas en las diferentes etapas que requiere la correcta adopción de una $\mathrm{CdS}$ en una organización. Una de las personas entrevistadas planteó la cuestión con toda claridad refiriéndose a 
su unidad administrativa: «(...) más allá del reparto de folletos con la CdS, no se ha producido una verdadera difusión, ni siquiera dentro del propio laboratorio, muchas personas que trabajan aqui desconocen su existencia o sólo la conocen gracias a la presentación oficial del Director. Además, mejor que sea así, porque de otra manera parte del personal podría reaccionar negativamente, piensan que les va a dar más trabajo y esas cosas (...)». En definitiva, el papel de las personas que forman parte de la organización es decisivo para dotar de sentido a las CdS, lo que se puede plantear en términos de necesidad de profundizar en el diálogo y consenso interno para su formulación o en la ampliación de su difusión y la implicación mayor del personal una vez implantadas.

En correspondencia con lo anterior, si bien se ha concretado una ausencia de cambios en la gestión del personal unidos a las CdS, no deja de ser cierto que ofrecen determinadas ventajas asociadas a esta labor. Las CdS se han citado como herramientas que colaboran en la modernización administrativa, la identificación de puntos débiles antes invisibles, el logro de una mayor implicación del personal en la actividad corriente o la clarificación de las funciones realizadas, antes difusas en la maraña administrativa. En este sentido, si bien quedan por cuantificar los resultados derivados de la implantación de las $\mathrm{CdS}$ y los posibles caminos a través de los que explorar sus capacidades para la mejora y el cambio de la gestión del personal de grandes organizaciones con miles de empleados, desde una perspectiva general, y dentro de las diferentes unidades o servicios con sus propias peculiaridades internas, de forma particular, no deja de ser cierto que los aspectos apuntados, entre otros, pueden facilitar estrategias de mejora interna en la gestión de personas, si se enfocan adecuadamente los problemas internos a solventar. En suma, la rigidez de los instrumentos de gestión de personal que caracterizan al sector público español no se solucionan de forma directa con CdS, sin embargo, éstas sí pueden ofrecer alternativas indirectas para la mejora, así como servir para concretar indicadores de los cambios en marcha, de manera que las personas dentro de las organizaciones sean capaces de enfrentarse a los mismos con mayor cantidad de información.

El balance respecto a los recursos materiales y tecnológicos viene a confirmar las líneas maestras del estudio. Si bien las CdS han contado con un fuerte impulso institucional, incluso normativo, desde el máximo nivel político y la unidad central encargada de la modernización administrativa en la Administración de la CAM, los diferentes servicios y unidades encargados de la implantación operativa no han compartido ese interés y, por tanto, no lo han traducido en nuevas dotaciones de recursos específicos para hacer frente a las necesidades de esta nueva herramienta de ges- 
tión. Lo que es más, el fuerte compromiso citado de la Administración autonómica parece que no ha previsto las necesidades concretas de recursos materiales o tecnológicos que requieren las $\mathrm{CdS}$ y no ha asumido las necesidades de financiación directa o indirecta, más allá de colaborar con la metodología de creación, la financiación de los folletos informativos o la organización de formación impartida en el Instituto Madrileño de Administración Pública.

En todo caso, el discurso modernizador asociado a las tecnologías parece que ha calado en cierta medida en referencia a las $\mathrm{CdS}$, considerando determinados incrementos en los recursos tecnológicos vinculados a su implantación. Si bien la adopción de las CdS ha conllevado la incorporación de nuevas herramientas tecnológicas, especialmente orientadas a la dotación de infraestructuras o software a medida, esto se ha solido hacer sin una clara reflexión sobre las verdaderas potencialidades y repercusiones de esa relación entre innovaciones tecnológicas y de gestión, lo que desvirtúa lo que podría considerarse como una relación inevitable, aunque no siempre bien aprovechada. Sin duda, se perciben algunas interacciones entre CdS y mejoras internas, sin embargo, no se terminan de concretar cambios sustantivos en las diferentes dimensiones de interés de la gestión pública, al menos, si nos dejamos guiar por los resultados obtenidos en la presente investigación. De hecho, en la mayoría de los casos, más que una transformación interna, existe un intento de remediar por todos los medios la perspectiva opaca que los ciudadanos suelen tener respecto de las organizaciones públicas.

\subsection{Balance general: el impacto de las Cartas de Servicios en la Administración de la Comunidad de Madrid: entre la continuidad y el cambio}

Como ha sido dicho, las CdS constituyen un instrumento que permite renovar los servicios públicos en una línea de exigencia y calidad, adoptando compromisos concretos ante los ciudadanos. No es objeto de nuestro análisis el impacto directo que la introducción de las mismas tiene sobre la ciudadanía, en sus diferentes manifestaciones, sino su capacidad transformadora y modernizadora de los servicios que las han adoptado. En otros apartados de este trabajo hemos tratado los escasos resultados en lo relativo a los recursos humanos y materiales, ahora vamos a realizar algunas reflexiones generales sobre el impacto intra-administrativo de la introducción de las Cartas a partir de los datos obtenidos de la investigación general que sobre Cartas de Servicios en la Administración de la Comunidad de Madrid hemos desarrollado recientemente. 
En primer lugar, respecto al diseño orgánico, la introducción de las CdS ha tenido un impacto muy limitado. Es verdad que se ha creado una dirección general de calidad y mejora de los servicios públicos, que apoya los procesos de elaboración y actualización de las cartas, y que además, particularmente en los inicios de su andadura, contó con un importante apoyo político, pero no lo es menos que, en opinión de algunos entrevistados, ese apoyo se habría difuminando con el tiempo y que, además, las responsabilidades en los servicios en lo relativo a la Carta no siempre son claras, no habiéndose en general institucionalizado procesos o grupos de mejora y actualización.

En segundo lugar, en lo relativo a los modos de gestión, no parece que la existencia en sí de la Carta haya variado mucho la dinámica de los servicios. La responsabilidad del cambio, cuando lo ha habido, se residencia en un mejor conocimiento y control de los procedimientos y procesos que derivaría no tanto de la introducción de las Cartas como de los cambios tecnológicos y, sobre todo, de la incorporación de la calidad como concepto matriz, del que serían deudores los instrumentos que necesita, siendo algunos de ellos, relacionados pero independientes entre sí, los manuales de procedimientos y las CdS. Esta falta de reconocimiento del impacto interno de las $\mathrm{CdS}$ sobre los procesos y procedimientos ha sido casi unánime entre el personal entrevistado, que daba mayor entidad a la introducción de la calidad en sí misma con la cultura que apareja.

En tercer lugar, y respecto a los recursos humanos y materiales, ya ha sido dicho que el impacto ha sido muy exiguo, y a lo escrito más arriba nos remitimos.

Y, finalmente, en lo referido a la generación de información y comunicación, aunque es uno de los aspectos «fuertes» de las CdS en su dimensión externa, no parece haber ocasionado cambios de interés a nivel interno más allá de haber definido determinados procedimientos de remisión y tratamiento de reclamaciones y sugerencias.

A la vista de lo anterior, y de acuerdo con los datos obtenidos en el trabajo de campo, cabe pensar que el mayor impacto de las CdS se ha realizado fuera de la Administración, en el terreno de la ciudadanía. En efecto, es unánime el reconocimiento que los servicios hacen de las $\mathrm{CdS}$ en tanto instrumento de comunicación de lo que se hace en la Administración, de la forma en que se hace, dentro de unos parámetros y compromisos específicos. En lo referido a este último aspecto, hay que reconocer que la generación de indicadores adecuados, que realmente permitan medir, junto a la asunción de compensaciones a favor del ciudadano en 
caso de no cumplirse lo comprometido, sigue siendo uno de los aspectos más débiles del modelo introducido en la Administración de la Comunidad de Madrid. Sólo algunas de las organizaciones públicas sometidas a una normativa procedimental próxima al mercado privado, es decir, las que constituyen la Administración instrumental de la Comunidad, han avanzado en este ámbito y merecen un reconocimiento aparte (por ejemplo, el Canal de Isabel II, o el Consorcio de Transportes de Madrid). Esto nos sugiere la necesidad de hacer una diferenciación entre las CdS vinculadas a los servicios prestacionales, regidos en el ejercicio de su actividad por normativa mercantil común y que tienen referentes o competidores externos (por ejemplo en el sector del transporte), que han avanzado razonablemente en la definición de CdS que plantean una suerte de «contrato» para el ciudadano, y las vinculadas a servicios destinados a la administración de potestades, regidos por la normativa administrativa y sin competencia externa, en los que las citadas CdS no difieren demasiado de una mera declaración de intenciones. Entre sendos extremos, se ubican el resto de los servicios, aunque es de esperar que con el tiempo y experiencia acumulados muchos de ellos paulatinamente se aproximen más al umbral de los primeros que al de los segundos.

Lo anterior, que demuestra palmariamente la diversidad material, procedimental y normativa en que se mueven nuestras Administraciones públicas, sin embargo no satisface del todo la pregunta de por qué, habida cuenta del potencial de la reforma que implica la calidad y las CdS, de las presiones del entorno para el cambio hacia la exigencia ciudadana, de una estructura de oportunidades favorable junto a un liderazgo reformador ambicioso y sólido, los resultados de la implantación de las CdS han sido relativamente paupérrimos. Una explicación radica en que la introducción de este tipo de herramientas es, por su propia naturaleza, lenta y gradualista. Pero también hay que considerar convenientemente la importancia de la cultura administrativa. Las herramientas de la calidad, y las $\mathrm{CdS}$ entre ellas, han demostrado una gran capacidad de adaptación a diversas culturas administrativas, pero flexibilizando sus perfiles de acuerdo con las necesidades y condicionantes administrativos de cada país. De esta forma, en los distintos países las cartas establecen una declaración de los servicios que se prestan, junto con estándares, indicadores de calidad mejor o peor perfilados y normas de acceso y atención a los ciudadanos. Las diferencias sin embargo, son notables en la concreción de los compromisos asumidos y el reconocimiento «efectivo» de derechos a favor del ciudadano en caso de no cumplirse. Pero es en aquellos países con una débil cultura burocrática, fundamentalmente anglosajones, donde los compromisos son más claros, concretos y susceptibles de provocar compensaciones para el ciudadano, es decir, donde los derechos de los 
ciudadanos frente a los servicios son más fuertes. Acaso sea porque las Administraciones en dichos países, y el caso de Reino Unido es paradigmático, se mueve en un entorno de autonomía de gestión muy amplia, de escasos controles procedimentales, con lo que la garantía jurídica frente al ciudadano debe articularse mediante otros instrumentos (las Cartas), frente al caso español y en general continental en que la actuación de los servicios públicos está muy regulada, también en lo procedimental, con un derecho ad hoc que garantiza los derechos de manera genérica y difusa. Esta explicación, plenamente aplicable a la Administración de la Comunidad de Madrid, se ve refrendada precisamente por el dato apuntado anteriormente, consistente en que la Administración instrumental, que deja mayor autonomía a los gestores y que se mueve en el derecho privado, es la que ha generado CdS en que los derechos de los ciudadanos se ven mejor recogidos y garantizados.

\section{CONCLUSIONES}

Las CdS son una de las herramientas de implantación de la calidad más utilizadas en las Administraciones públicas españolas, así como de otros países de nuestro entorno. Debido a las lagunas en el estudio de las CdS en nuestro país, se desarrolló una investigación, en la que participaron los autores, sobre los efectos de su implantación dentro de una Administración concreta, de manera que este trabajo ofrece parte de los resultados de un estudio más amplio sobre la Comunidad de Madrid y, dentro de ella, en referencia al impacto interno en lo concerniente a los recursos humanos, materiales y tecnológicos. En general, los datos han mostrado que las $\mathrm{CdS}$ han tenido un impacto relativamente exiguo en las dimensiones de análisis. Por un lado, en lo que respecta al capital humano, la participación de los distintos tipos de personal en el diseño, implantación y evaluación de las CdS ha sido importante en el ámbito directivo e, incluso, del personal operativo, aunque en menor medida, mientras que su influencia ha sido prácticamente nula en las diferentes tareas de gestión del personal (acceso, promoción, retribución, modificación del contenido de los puestos, motivación y sistema del reconocimiento), salvo en el ámbito de la formación. Por otro lado, en lo que se refiere a los recursos materiales $\mathrm{y}$ tecnológicos, los datos sugieren que las $\mathrm{CdS}$ han permitido determinados gastos extraordinarios, fundamentalmente en el plano tecnológico, mientras las dimensiones humana y material han quedado prácticamente relegadas de esos aumentos. También se puso de manifiesto que las CdS, al menos de forma mínima, requieren una combinación con nuevas aplicaciones informáticas o tecnológicas, bien para explorar su dimensión de 
atención ciudadana, bien para mejorar su dimensión de gestión interna. En definitiva, a pesar de que los resultados son limitados, la Administración de la Comunidad de Madrid ha intentado asimilar las experiencias desarrolladas en otros contextos y se ha convertido con ello en pionera en el ámbito autonómico de nuestro país, a pesar de que las inercias burocráticas e institucionales han sido factores condicionantes del rendimiento del modelo. Aunque el análisis específico de dichos factores va más allá de los objetivos de este artículo.

En conclusión, las CdS tienen que hacer frente a determinadas barreras para su puesta en marcha pero, una vez adoptadas, disponen de una serie de potencialidades para las organizaciones administrativas que las desarrollan. Los aspectos analizados en este estudio ponen de manifiesto la necesidad de tener en cuenta el contexto institucional concreto en el que se insertan las CdS. Más allá de esquemas del tipo todo tiempo y lugar, este segmento de herramientas requieren de una aproximación a medida, que permita una efectiva y progresiva adopción y difusión en unidades y servicios concretos, teniendo en cuenta la variedad de sub-culturas existentes en grandes organizaciones como la Administración de la Comunidad de Madrid. Algunos casos que han incorporado una CdS han identificado ciertos resultados unidos a estas herramientas que, si bien suelen adolecer de una perspectiva general sobre la organización y son escasamente generalizables al resto, al menos ofrecen algunos puntos de mejora derivados de su implantación, sobre todo en cuanto a los recursos tecnológicos disponibles, que ya están en marcha y que justifican los esfuerzos e inversiones realizadas.

\section{BIBLIOGRAFÍA}

Albi, Emilio, (2000): Público y Privado. Un Acuerdo Necesario. Barcelona: Ariel.

Ballart, Xavier, (2001): Innovación en la Gestión Pública y en la Empresa Privada. Madrid: Díaz de Santos.

BALlaRT, Xavier (1996): «Indicadores para el control de la gestión de personal», en Gestión y Análisis de Políticas Públicas, n. ${ }^{\circ}$ 5-6, pp. 101 y ss.

Bazaga, Isabel, Ernesto Carrillo, Juan A. Ramos, José Sosa, y Manuel TAmayo, (1998): El Consumo y la Calidad de los Servicios Públicos. Estudio de Caso de la Ciudad de Coslada. Madrid: Consejería de 
Medio Ambiente y Desarrollo Regional, Comunidad de Madrid-Ayuntamiento de Coslada.

Bynoe, Ian, (1996): Beyond the Citizen's Charter: New Directions for Social Rights. London: Institute for Public Policy Research.

Chandler, J. A. ed. (1996), The Citizen's Charter. Aldershot: Dartmouth.

CRESPO GONZÁLEZ, Jorge (2001): La implantación de un sistema de atención al ciudadano a través de la calidad total en un Ayuntamiento: la gestión del proceso, en Revista de Estudios Locales (Cunal).

Criado, J. Ignacio, (2004): Construyendo la e-Administración Local. Las Tecnologías de la Información y la Comunicación e Internet en los Ayuntamientos de la Comunidad de Madrid. Madrid: EuroGestión Pública.

Dirección General de Calidad de los Servicios, (1998): Manual de Implantación del Plan de Calidad de los Servicios de la Comunidad de Madrid. Madrid: Consejería de Hacienda, Comunidad de Madrid.

Gómez Rivas, José V. (2004): Gobernanza Democrática en el Nivel Local. De la Gestión de Puestos a la Gestión del Conocimiento. Valencia: Diputación de Valencia.

LóPez, Jordi, y Albert GAdEA, (2001): Una Nueva Administración Pública. Estrategias y Métodos para Mejorar la Calidad y la Eficiencia del e-Gobierno. Oñati: Instituto Vasco de Administración Pública.

LóPeZ, Guillem, ed. (2003): Los Nuevos Instrumentos de la Gestión Pública. Barcelona: Caja de Ahorros y Pensiones de Barcelona.

McGuire, L. (2001): «Service Charters-Global Convergence or National Diverge? A Comparison of Initiatives in Australia, the United Kingdom and the United States». Public Management Review 3 (4):493-524.

Moreno, José Luis, (1998): «Implantación del Plan de Calidad en la Comunidad de Madrid (1995-1998)». Gestión y Análisis de Políticas Públicas 11-12:61-81.

OlíAs de Lima, Blanca, (2002): «Las Administraciones Públicas Españolas: Balance y Perspectivas». En Administraciones Públicas Españolas, eds. J. Crespo y G. Pastor. Madrid: McGraw-Hill, pp. 367-393. 
Olías de LimA, Blanca, (2003): «La Mejora de los Servicios Públicos y la Revisión de las Relaciones entre la Administración y el Ciudadano: las Cartas de Servicios». Reforma y Democracia 25.

Pollit, Christopher, (1994): «The Citizen's Charter: a Preliminary Analysis». Public Money \& Management 14 (2):9-14.

Pollit, Christopher, y Stephen Harrison, eds. (1992): Handbook of Public Services Management. Oxford: Blackwell.

Sancho, David, (1999): Gestión de Servicios Públicos: Estrategias de Marketing y Calidad. Madrid: Tecnos-Universitat Pompeu Fabra.

SHARMA, Alok y ViveK K. Agnihotri, (2001): «The Citizen's Charter: the Indian Experience» International Review of Administrative Sciences 67 (4):733-740.

Schiavo, Luca, (2000): «Quality Standards in the Public Sector: Differencies between Italy and the UK in the Citizen's Charter Initiatives»». Public Administration 78 (3):679-698.

Willett, Chris, (1996): ed. Public Sector Reform and the Citizen's Charter. London: Blackstone Press. 